\title{
Research on De-icing Robot and Its Motion Characteristics Overhead Transmission Line
}

\author{
Zhuo Wang ${ }^{1}$, Ming-zhi Cai ${ }^{1}$, Tao Wang ${ }^{2}$ and Bo Zhang ${ }^{1, *}$ \\ ${ }^{1}$ College of Mechanical and Electrical Engineering, Harbin Engineering University, Nantong Street 145,150001 Harbin, \\ China \\ ${ }^{2}$ School of MechanicalEngineering, Hebei University of Technology, 300401 Tianjin, China \\ Corresponding Email: jlwzh@163.com
}

\begin{abstract}
In recent years, most areas of our country are suffering transmission line ice-coating disasters caused by cold weather. Aiming at this phenomenon, this paper designs a robot that not only can avoid the various fittings on the line, but also can cross the tension tower of phase line to jumper line. According to the development status of the deicing robot at home and abroad, the specific environment of the transmission line, as well as the function of crossing obstacle and tension tower, the design specifications and structural scheme is presented. On the basis of the mechanical structure design, the motion characteristics of deicing robot is researched, and the virtual prototype of the robot is simulated based on ADAMS, and key research is the factors which affect the gravity center when the robot crosses obstacle and tower. With the analysis of factors mentioned, robot stability greatly improved.
\end{abstract}

\section{Introduction}

The ice on overhead transmission lines seriously affects the normal working of the grid and even brought a huge disaster [1]. The icing on the insulators and conductors ice cause flash trip conductor galloping, equipment damage, when the thickness of the ice cover significantly beyond the carrying capacity of the line, the ice accident such as tower down, lines break and the large blackouts will happen [2]. However, strategic assets such as transmission grids need to be operated in a safe, predictable, and reliable way. In light of this, in order to reduce the hazards of rain and snow on the transmission system and protect the safe operation of the power grid [3, 4], the design of effective de-icing tool to remove ice on the transmission cable, has important practical significance. Foreign scholars, such as Japan scholars, Institute de Recherché hydro-Québec (IREQ), as well as Portugal and Mexican scholars have done some research on the deicing robot, and a lot of domestic universities and institutes have also done the research [5-7].

\section{Design requirements and overall scheme}

\subsection{Overhead transmission lines}

The transmission line is mainly composed by the conductor, insulator strings, line fittings, tower, and guy, basic and grounding devices. There is also installed lightning conductor on the high overhead transmission lines. Insulator strings, high-altitude warning bell, and a variety of fittings such as suspension clamp, damping hammer and spacing rods, shown in the Figure1, these relative de-icing robot is a variety of obstacles. Therefore, the robot must have the capability to cross obstacles [8].

The strain section of the lines is mainly constituted by straight type tower and strain type tower. Straight type tower plays the role of supporting and fixing the cable without tension, while the strain type tower support cable with the tension between the wires and conductor gravity. The strain section of the lines is shown in the Figure2. Therefore, in order to continuously working between towers, the deicing robot must have the ability to cross towers [9]. 


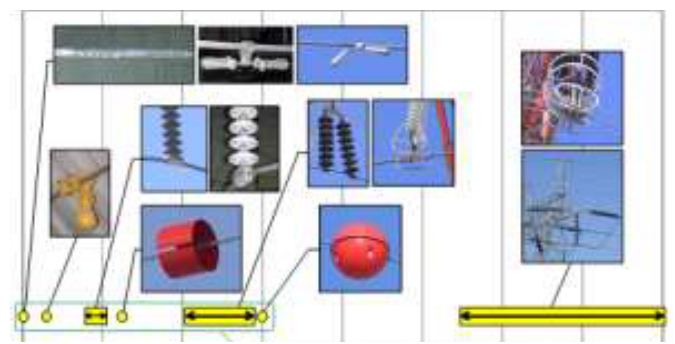

Fig. 1. Obstacles classified by length.

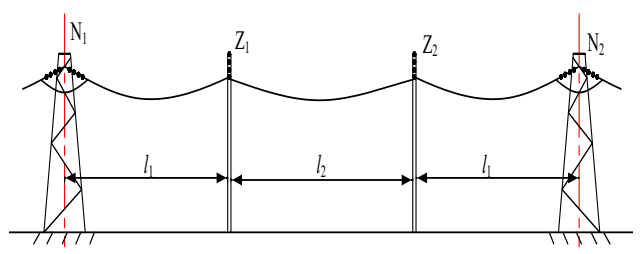

Fig. 2. A strain segment of the line.

\subsection{Design constraints and technical specifications}

The robot works on the long transmission lines at $15-30 \mathrm{~m}$ high, where the environmental conditions are harsh. Firstly, to improve efficiency, the robot need to walk and deicing stable, and has the ability of cross the obstacles and towers. Meanwhile, in order to long adequate work, the robot is required light and small and low power consumption. And the environment and technical specifications of the robot are shown in the following Table 1.

Table 1. Design specifications.

\begin{tabular}{|c|c|}
\hline \multicolumn{2}{|c|}{ Environmental specifications } \\
\hline Voltage level & $110 \mathrm{kV}$ \\
\hline Conductor diameter & $\begin{array}{c}12 \mathrm{~mm}- \\
50 \mathrm{~mm}\end{array}$ \\
\hline Maximum slope in span & $30^{\circ}$ \\
\hline Maximum obstacle diameter & $200 \mathrm{~mm}$ \\
\hline Maximum obstacle length & $760 \mathrm{~mm}$ \\
\hline Maximum wind level & 7 \\
\hline \multicolumn{2}{|c|}{ Robot specifications } \\
\hline Weight & $40 \mathrm{~kg}$ \\
\hline Traction force & $500 \mathrm{~N}$ \\
\hline Inspection speed & $1.0 \mathrm{~m} / \mathrm{s}$ \\
\hline Deicing speed & $3.0 \mathrm{~m} / \mathrm{min}$ \\
\hline Battery life & $5.0 \mathrm{hours}$ \\
\hline Telecommunication signal range & $5.0 \mathrm{~km}$ \\
\hline
\end{tabular}

\subsection{Mechanical structure schemes and working principle}

The robot is composed by a pair of deicing mechanism, a pair of driving mechanism, a pair of clamping mechanism, a pair of support mechanisms, and a control box. Through the motor base of driving mechanism, the deicing mechanism and clamping mechanism are fixed on driving mechanism. The deicing mechanism is fixed in the front, used to get rid of the ice coating before the robot, and the mechanism is fixed in the post, when the robot crossing obstacles and brake. The motor base of driving mechanism is fixed on the top of the support mechanism, and two support mechanisms are fixed in two sliding arms of the control box, which can slide and pitch relative by each other.

Joints and mechanical structure scheme of the robot are shown in Figure3.

The working process of the robot includes avoid obstacles action, across obstacles action, drop back action, clamp and release cables action, and cross towers action. The following is specifically described. 
(1)Avoid obstacles action. Through a pair of bevel gears, the arm motor drives the up-arm swing relative to downarm along a certain angle, so as to make the driving mechanism swing away from cables.

(2)Across obstacles to action. Through a series of racks and pinions in control-box, box motor drive two arms slide relative, so as to make the driving mechanism pass obstacles.

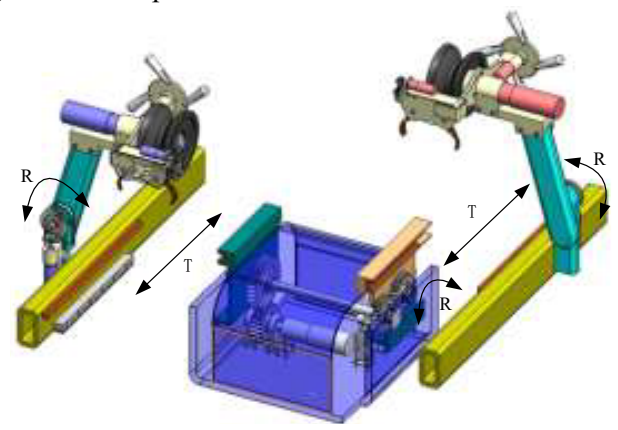

Fig. 3. Mechanism and movement joints of the deicing robot.

(3)Drop back action. Similar like the avoid obstacles action, the drop back action is the inverse process of avoiding obstacles action.

(4)Clamp and release cables action. In order, to ensure the stability of the robot, when one driving mechanism crosses obstacles, the clamping mechanism on another driving mechanism will clamp the cable. In this case, the motor in clamping mechanism will drive two clamping hands clamp the cable through the gears and turbine worms, so as to achieve clamp and release cables action.

(5)Across towers action. When the robot is walking to the tension tower end, it is required to cross the tower from the phase line to the leading line, and it has a certain angle between the phase line and the leading line. At this time, through the worms in the control box, the motor drives the arm rotates relative to the box, so as to achieve across towers action.

\section{Motion characteristics}

\subsection{The deduction of connecting rod coordinates system and its motion equation}

There're a total of five in deicing robot joints, respectively, $T_{1}, T_{2}, R_{l}, R_{2}$ and $R_{3}$, as showed in Figure 3. In Figure 4, the joints 2 and 6 for mobile vices, joints, 3,5 , and 7 for the rotation.

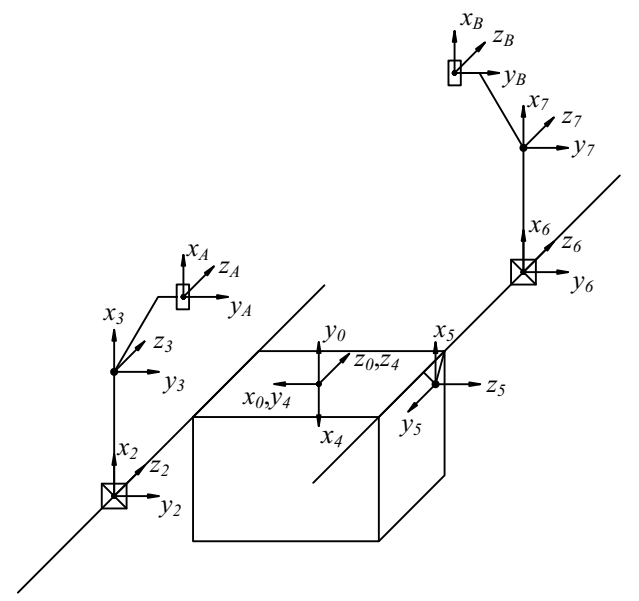

Fig. 4. B bracket each link coordinates system.

Set up between two connecting rods $i-1$ and $i$ by two rotations and translation after the prescribed the coordinates of all connecting rod. This relationship is described by four homogeneous transformations. This relation $A_{i}$ matrix is:

$$
A_{i}=\operatorname{Rot}\left(z, \theta_{i}\right) \operatorname{Trans}\left(0,0, d_{i}\right) \operatorname{Trans}\left(a_{i}, 0,0\right) \operatorname{Rot}\left(x, \alpha_{i}\right)
$$

Open type is obtained: 


$$
A_{i}=\left[\begin{array}{cccc}
c \theta_{i} & -s \theta_{i} c \alpha_{i-1} & s \theta_{i} s \alpha_{i-1} & a_{i-1} c \theta_{i} \\
s \theta_{i} & c \theta_{i} c \alpha_{i-1} & -c \theta_{i} s \alpha_{i-1} & a_{i-1} s \theta_{i} \\
0 & s \alpha_{i-1} & c \alpha_{i-1} & d_{i} \\
0 & 0 & 0 & 1
\end{array}\right]
$$

For prismatic joints, $A$ matrix is:

$$
A_{i}=\left[\begin{array}{cccc}
c \theta_{i} & -s \theta_{i} c \alpha_{i-1} & s \theta_{i} s \alpha_{i-1} & 0 \\
s \theta_{i} & c \theta_{i} c \alpha_{i-1} & -c \theta_{i} s \alpha_{i-1} & 1 \\
0 & s \alpha_{i-1} & c \alpha_{i-1} & d_{i} \\
0 & 0 & 0 & 1
\end{array}\right]
$$

Connecting rod available transformation is given as follows:

$$
{ }^{i-1} T_{1}=\left[\begin{array}{cccc}
c \theta_{i} & -s \theta_{i} & 0 & a_{i-1} \\
s \theta_{i} c \alpha_{i-1} & c \theta_{i} c \alpha_{i-1} & -s \alpha_{i-1} & -d_{i} s \alpha_{i-1} \\
s \theta_{i} s \alpha_{i-1} & c \theta_{i} s \alpha_{i-1} & c \alpha_{i-1} & d_{i} c \alpha_{i-1} \\
0 & 0 & 0 & 1
\end{array}\right]
$$

Where $s$ is sin, $\mathrm{c}$ is $\cos , \theta_{\mathrm{i}}$ is two connecting rod angle, $\alpha_{\mathrm{i}-1}$ is connecting rod torsion angle, $a_{\mathrm{i}-1}$ is connecting rod base tangent length, $d_{\mathrm{i}}$ is two connecting rod distance.

According to the above principles, deicing robot $A$ bracket connecting rod parameters is shown in Table 2 .

Table 2. Deicing robot linkage parameters of $A$ bracket

\begin{tabular}{|c|c|c|c|c|}
\hline Connecting rod $\boldsymbol{i}$ & $\boldsymbol{\theta}_{\mathbf{i}}$ & $\boldsymbol{\alpha}_{\mathrm{i}-\mathbf{1}}$ & $\boldsymbol{a}_{\mathbf{i}-\mathbf{1}}$ & $\boldsymbol{d}_{\mathbf{i}}$ \\
\hline 1 & $0^{\circ}$ & $0^{\circ}$ & 0 & $d_{1}$ \\
\hline 2 & $-90^{\circ}$ & $0^{\circ}$ & $a_{1}$ & $d_{2}$ \\
\hline 3 & $\theta_{3}=0^{\circ}$ & $0^{\circ}$ & $a_{2}$ & \multicolumn{2}{|c|}{0} \\
\hline
\end{tabular}

The coordinate system A observed changes in the matrix of $T_{\mathrm{A}}$ :

$$
T_{A}=T_{3}^{3} T_{A}={ }^{0} T_{1}^{1} T_{2}^{2} T_{3}^{3} T_{A}=\left[\begin{array}{cccc}
s_{3} & c_{3} & 0 & a_{1}+L c_{3}+a_{2} s_{3} \\
-c_{3} & s_{3} & 0 & -a_{2}-a_{2} c_{3}+L s_{3} \\
0 & 0 & 1 & d_{1}+d_{2} \\
0 & 0 & 0 & 1
\end{array}\right]
$$

In the same way, the robot B bracket each link coordinates system is established. Change the matrix of A relative to B ${ }^{B} T_{A}$

$$
{ }^{B} T_{A}={ }^{B} T_{0}^{0} T_{A}=\left({ }^{0} T_{B}\right)^{-1} \cdot{ }^{0} T_{A}
$$

The change of B relative to A matrix of the ${ }^{A} T_{B}$

$$
{ }^{A} T_{B}={ }^{A} T_{0}^{0} T_{B}=\left({ }^{0} T_{A}\right)^{-1} \cdot{ }^{0} T_{B}
$$

\subsection{Cross obstacles process}

Save the Solid works model of robot as para solid x_t file, and import it into ADAMS. Definitely the material or weight of all parts, and add constraints, drives, and forces to the robot model. Then set the contact impact parameters, verify the model, and take the simulation analysis.

Choose the Cartesian coordinate system in ADAMS model space, in which the z-axis is the forward direction of the robot, and the $\mathrm{y}$-axis is the opposite direction of gravity. The clamping mechanism on arm B clamps cable before arm A awing. The rotation joint between up-arm and down-arm is the rotation center of the arm. Then the arm swings to side and the driving wheel out of cable, the curves are shown in Figure 5(a)-(d). 


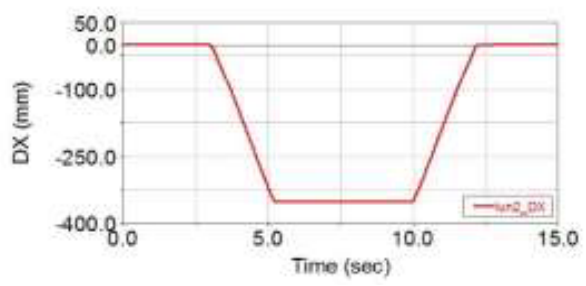

(a)

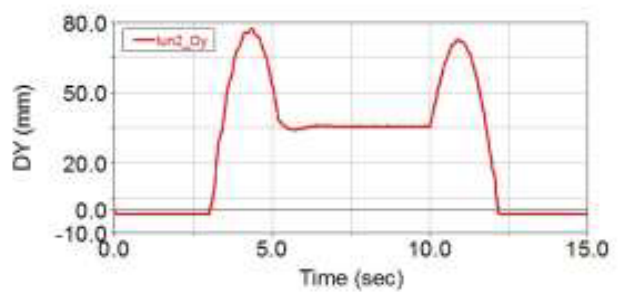

(b)

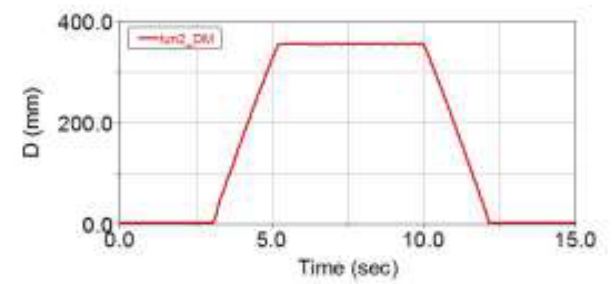

(c)

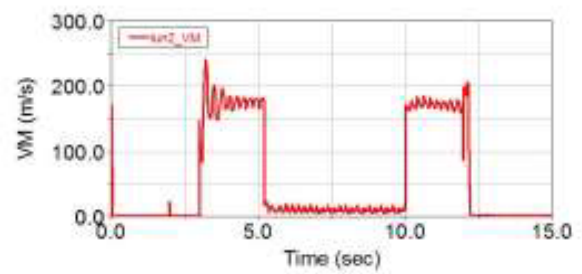

(d)

Fig. 5. The curve of the $\mathrm{x}$-axis, $\mathrm{y}$-axis, the displacement and velocity on driving wheel $\mathrm{A}$.

The curve of the $\mathrm{x}$-axis on driving wheel $\mathrm{A}$ is shown in Figure 5(a).The curve of the $\mathrm{y}$-axis on driving wheel $\mathrm{A}$ is shown in Figure5 (b), the curve of the displacement on driving wheel A is shown in Figure 5(c), the curve of the velocity on driving wheel A is shown in Figure5(d).

From the Figure5 (a), (b), (c), we can get that the clamp mechanism B clamps cable at 2 seconds, and begin to swing at 3 seconds, and swings to the maximum position at 5.2 seconds with the role of the positioning pins. As is shown in the Figure $4(\mathrm{~d})$, the speed is vibration changes in the swing process, due to the change of robot gravity center caused by driving wheel swinging. Throughout the swing and drop back action, of the wheel speed has obvious mutation at 3 seconds, 5.2 seconds, 10 seconds and 12.2 seconds. The change of gravity center of the robot, caused by the swinging of the arm A, is shown specifically in Figure6(a),(b).

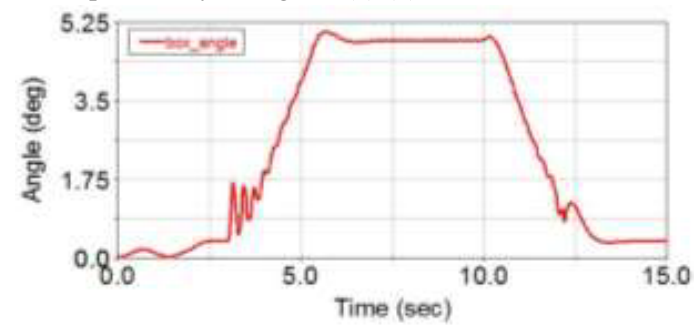

(a) 


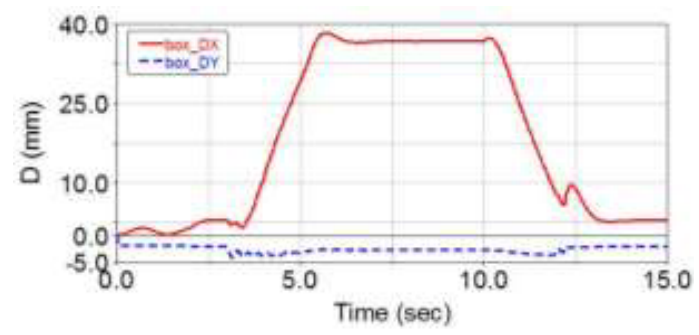

(b)

Fig. 6. The curve of eccentric angle $\alpha$ and the coordinates of the gravity center.

The curve of eccentric angle $\alpha$ and the coordinates of the gravity center is shown respectively in Figure 7. As showed in Figure7, eccentric angle $\alpha$, the deflection angle of the robot relative to the central axis, is increasing gradually with driving wheel A swinging side, and reaches a maximum of 5.2 in 5.2 seconds. When the arm A starts to swing back to 10 seconds, the centroid of the entire robot gets back gradually again, so that the driving wheel A can drop back to the cable opportunely.

After the arm A swing side, two arms slides on the control box relative, and then the arm A drop back on the cable. During this crossing obstacles process, the curve of the gravity center of coordinates changed during crossing obstacle is shown in Figure7.

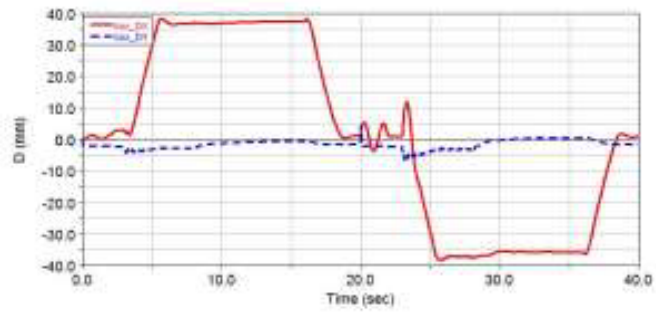

Fig. 7. The curve of the gravity center during crossing obstacle.

As is shown in Figure7, the centroid of robot changes little by its gravity before the arm A swinging. But when the arm A begins swinging to side in 3 seconds, the centroid $\mathrm{m}$ of the entire robot change significantly along the $\mathrm{x}$-axis from 0 to $40 \mathrm{~mm}$, and gradually, the arm A get re-balanced at another side.

The mass center of the entire robot, when the arm A swing back again $(t=18 \mathrm{~s})$, gradually returns to its original position, and then the driving wheel A down to the cable. In the process of the relative movement of the two sliding $\operatorname{arms}(5.2 \mathrm{~s}, 18 \mathrm{~s})$ and $(25 \mathrm{~s}, 38 \mathrm{~s})$, the center of gravity is stable. Similarly, the gravity center swing with the swinging of the arm B. In the whole crossing obstacles process, the gravity center of robot changes very litter along the $y$-axis, in the range of $0-0.5 \mathrm{~mm}$.

The trajectory of driving wheel A, B and gravity center during crossing obstacle is shown in Figure8. As is described in Figure8, with the swing of the arm, the gravity center of robot offset to another side to make re-balance on the cable.

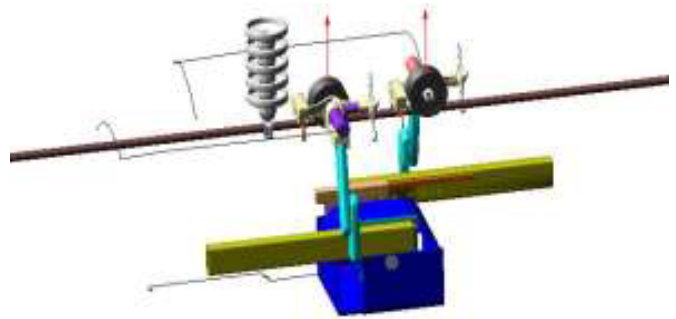

Fig. 8. The trajectory of driving wheel A, B.

\subsection{Crossing towers process}


When the robot has completed the de-icing task to the end of the cable, it needs across the tower. If it is straight tower, the robot can cross it through the crossing obstacles action, or it is strain type tower, the robot needs to transfer the path from phase line to the drainage line, so as to achieve continuous working between towers.

In the crossing towers process simulation, the gravity center of the robot also changed due to swinging of the arm, so that the robot can get to rebalance. The gravity center is offset along $\mathrm{x}$-axis in the range of $-40 \mathrm{~mm}$ to $40 \mathrm{~mm}$, as is shown in Figure9.

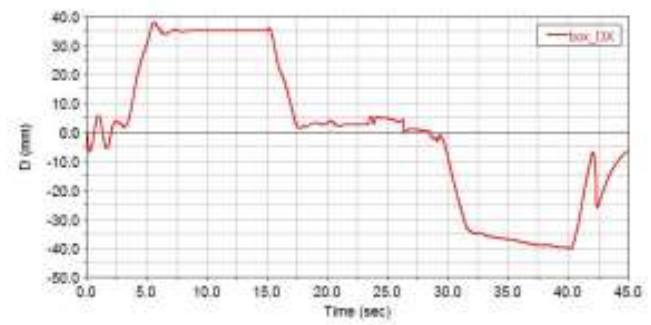

Fig. 9. The curve of gravity center during crossing tower.

Figure 10 depicts the trajectory of two drive wheels and gravity center during crossing tension tower. With the swing of the arm, the gravity center of robot offset to another side to make re-balance on the cable.

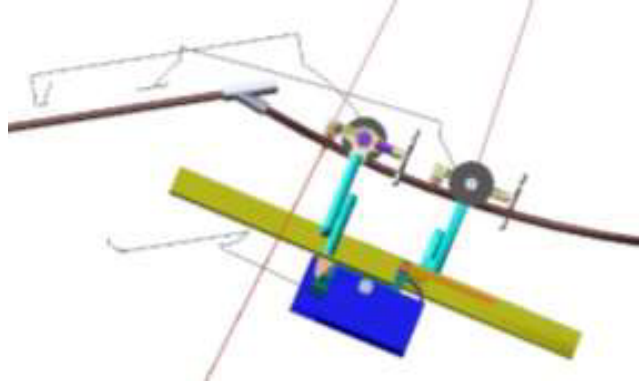

Fig. 10. The trajectory of two drive wheels.

\subsection{Stability improvements}

Through the crossing obstacles process and crossing towers process simulation, the position of rotation joint between the up-arm and the down-arm affect the driving wheels' swinging up action and drop back action, due to the wheel flange may collision with cable even cannot drop back.

The factors causes the gravity center offset are mainly constituted by following: 1) the mass ratio between the driving mechanism and the control box, 2) the swing range and swing speed of the driving wheel, 3) the clamping force of the clamping mechanism. Based on the points above, by adjusting the swing angle of the arm to $45^{\circ}$, adjusting the final mass of the control box to $25 \mathrm{~kg}$ in order to increase the mass ratio between the control box and the driving mechanism, while making the gravity center of the robot just below the cable; increased to intensify the coefficient of friction between the institutions and cable, increases the friction coefficient between the clamping mechanism and the cable.Figure11.

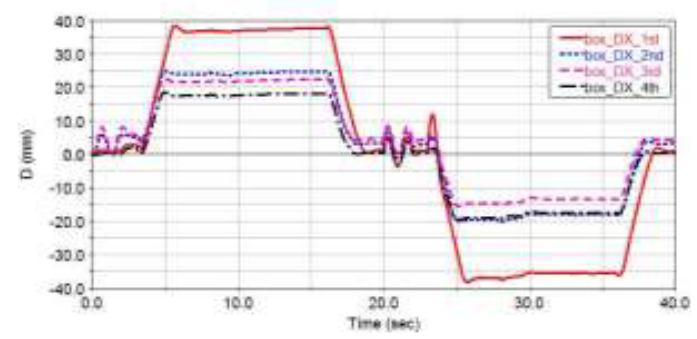

Fig. 11. The curve of the angle during crossing obstacle.

Figure12 depicts the curve of the eccentric angle and coordinate of the gravity center during crossing obstacle and tension tower. 


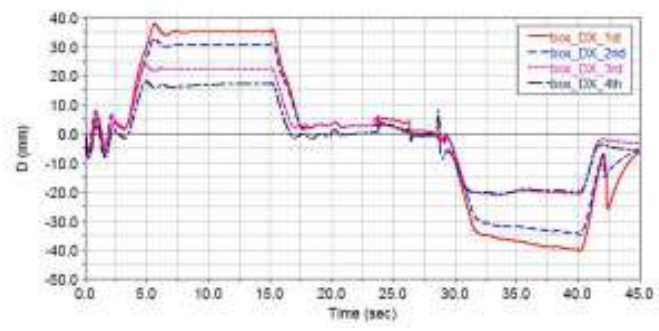

Fig. 12. The curve during crossing tension tower.

\section{Conclusion}

The coordinate system for the deicing robot was established at first, and deduced the kinematics equation. Then the virtual prototype model of deicing robot in kinematics simulation was analyzed. Deicing robot based on the simulation results can be obtained can smooth cable on the barrier; verify the correctness of the model. Factors influencing the robot center of gravity change was obtained by the simulation and it can be mainly composed of the following factors:

(1)The quality ratio between the driving mechanism and the control box;

(2) The location of the robot center of gravity;

(3) The driving mechanism of swing range and the size of the swing speed;

(4) The clamping force of clamping device. By adjusting the above factors, it can make the robot more stable cross obstacle or crossing tower, to improve the stability of the robot.

\section{Acknowledgement}

This material is based upon work supported by the National Science Foundation of China (Grant no. 51479043). The views expressed are authors alone.

\section{References}

1. K. Toussaint, N. Pouliot, S. Montambault. J Field Robot, 26,5 (2009)

2. R. I. Egbert, R. L. Schrag, W. D. Bernhart, et al. IEEE T Power Deliver, 4, 3 (1989)

3. K. Toussaint, N. Pouliot, S. Montambault. J Field Robot Wiley, 9 (2009)

4. J Michael, T. Moser, K. Bretter. IEEE SENS J, 35,2 (2010)

5. T.G. Brown. Cold Regions Science and Technology, ( 2007)

6. H. Zangl, T. Bretterklieber, and G. Brasseur.IEEE T Instrum Meas, 58,5 (2009)

7. S. Montambault, N. Pouliot. The HQ Line ROVer: contributing to innovation in transmission line maintenance, (2003)

8. M. J. Moser, T. Bretterklieber .IEEE T Ind Electron, 58,7 (2011)

9. J. Sawada ,K. Kusumoto, Y. Maikawa, et al. IEEE T Power Deliver. 6,1 (1991) 